Pacific Journal of Mathematics

COHOMOLOGY OF ACTIONS OF DISCRETE GROUPS ON 


\title{
COHOMOLOGY OF ACTIONS OF DISCRETE GROUPS ON FACTORS OF TYPE $\mathrm{II}_{1}$
}

\section{YASUYUKI KAWAHIGASHI}

\begin{abstract}
We study 1-cohomology of discrete group actions on factors of type $I_{1}$. Characterizations of Kazhdan's property $T$ and amenability for discrete groups in terms of cocycles and coboundaries are given, and we show that each of $\operatorname{SL}(n, \mathbf{Z}), n \geq 3$, and $\operatorname{Sp}(n, \mathbf{Z}), n \geq 2$, has a continuous family of mutually non-cocycle conjugate free actions on the AFD factor of type $\mathrm{II}_{1}$ as an application. We also introduce and compute entropy for discrete amenable group action on factors of type $\mathrm{II}_{1}$ •
\end{abstract}

0. Introduction. In this paper, we study 1-cohomology of discrete group actions on factors of type $\mathrm{II}_{1}$. We give characterizations of Kazhdan's property $T$ and amenability for discrete groups in terms of 1-cocycles and coboundaries for actions on factors of type $\mathrm{II}_{1}$. As an application, we also show that each of $\operatorname{SL}(n, \mathbf{Z}), n \geq 3$, and $\operatorname{Sp}(n, \mathbf{Z}), n \geq 2$, has a continuous family of mutually non-cocycle conjugate ergodic free actions on the approximately finite dimensional (AFD) factor of type $\mathrm{II}_{1}$. These are typical groups with Kazhdan's property $T$. We introduce and compute entropy of discrete amenable group actions on the AFD factor of type $\mathrm{II}_{1}$.

Complete classification of actions of discrete amenable groups on the AFD factor $\mathscr{R}$ of type $\mathrm{II}_{1}$ up to cocycle conjugacy was given in Ocneanu [O]. In particular, he showed that any two free actions of a discrete amenable group on $\mathscr{R}$ are cocycle conjugate. Then Jones [J2] showed that this statement is no longer valid for any discrete nonamenable group. He constructed two free actions and used the ergodicity at infinity to distinguish the two. This shows that non-amenable discrete groups are quite different from amenable ones in the theory of group actions on factors. In order to understand cocycle conjugacy of nonamenable group actions, we start to study 1-cohomology of the actions and get several von Neumann algebra analogues of Schmidt's work [S] on ergodic actions on probability spaces. A major difference between the cohomology theory on probability spaces and one on von Neumann algebras is that we do not have the group structure on the space of cocycles in the latter case, which causes technical difficulty. 
First we work on groups with Kazhdan's property T, which are far from being amenable. (See $\S 1$ for the definition.) Since Connes [C3], several authors have shown that the discrete groups with Kazhdan's property $\mathrm{T}$ are the opposite extreme of amenable groups with respect to representations and actions on probability spaces and von Neumann algebras. Here a characterization of Kazhdan's property $\mathrm{T}$ in terms of cocycles and coboundaries is given in $\S 1$. Jones [J1] showed two mutually non-cocycle conjugate free actions of discrete groups with Kazhdan's property $\mathrm{T}$ on the AFD factors of type $\mathrm{II}_{1}$, and $\mathrm{M}$. Choda [Ch2] showed four of such actions. We exhibit a continuous family of mutually non-cocycle conjugate free actions on the AFD factor of type $\mathrm{II}_{1}$ for certain groups with Kazhdan's property $\mathrm{T}$ like $\mathrm{SL}(n, \mathrm{Z}), n \geq 3$ and $\operatorname{Sp}(n, \mathbf{Z}), n \geq 2$, using her construction in [Ch2]. These cannot be distinguished by ergodicity at infinity as in Jones [J2]. Instead, we make use of the rigidity argument of cocycles to show that the "almost all" pair in the family is not mutually cocycle conjugate. This shows another aspect of rigidity in operator algebras. (See Connes [C3], Connes-Jones [CJ1, CJ2].)

In $\S 2$, we work on amenable groups. We introduce and compute Connes-Størmer entropy for discrete amenable group actions on the AFD factor of type $\mathrm{II}_{1}$ to distinguish continuously many non-commutative Bernoulli shifts. Then we get a characterization of amenability in terms of cocycles and coboundaries, based on Ocneanu's work [O]. This shows another remarkable difference between the discrete groups with Kazhdan's property $\mathrm{T}$ and discrete amenable groups.

The author is thankful to Professor M. Choda for helpful conversations and to Professor M. Takesaki for a suggestion improving the exposition.

1. Kazhdan's property $\mathbf{T}$ and cohomology classes. Let $G$ be a discrete (countable) group and $\alpha$ an action of $G$ on a factor $\mathscr{M}$ of type $\mathrm{II}_{1}$. Let

$$
\begin{aligned}
Z_{\alpha}^{1}=\left\{u: G \rightarrow \mathscr{U}(\mathscr{M}) \mid u_{g} \alpha_{g}\left(u_{h}\right)=u_{g h}, g, h \in G\right\}, \\
B_{\alpha}^{1}=\{u: G \rightarrow \mathscr{U}(\mathscr{M}) \mid \text { there exists } v \in \mathscr{U}(\mathscr{M}) \\
\text { such that } \left.u_{g}=v \alpha_{g}\left(v^{*}\right)\right\} .
\end{aligned}
$$

Topology of $Z_{\alpha}^{1}$ is given by the strong convergence at each $g \in G$. This topology is given by the following metric:

$$
d(u, v)=\sum_{n=1}^{\infty} \frac{1}{2^{n}}\left\|u_{g_{n}}-v_{g_{n}}\right\|_{2}, \quad \text { where } G=\left\{g_{n} \mid n \geq 1\right\}
$$

Consider the induced metric on $B_{\alpha}^{1}$. 
We say two cocycles $u_{g}, v_{g}$ are cohomologous and write $u_{g} \sim v_{g}$ if there exists a unitary $u \in \mathscr{M}$ such that $u_{g}=u v_{g} \alpha_{g}\left(u^{*}\right)$. Let $H_{\alpha}^{1}=Z_{\alpha}^{1} / \sim$ be the set of cohomology classes. Araki-Choda [ACh] called an action $\alpha$ strong if any asymptotically $\alpha$-fixed sequence in $\mathscr{M}$ is equivalent to a bounded sequence in $\mathscr{M}^{\alpha}$ and showed that a discrete group has Kazhdan's property $T$ if and only if every action of it on a factor of type $\mathrm{II}_{1}$ is strong. This is a non-commutative analogue of Connes-Weiss [CW] to the effect that a discrete group has Kazhdan's property $\mathrm{T}$ if and only if every ergodic measure-preserving action of it on a probability space is strongly ergodic.

We show that strong actions have a certain good property about cocycles. The following technique is based on that of Connes [C1, Theorem 3.1], and we note an analogy between central sequences and asymptotically $\alpha$-fixed sequences.

Proposition 1. If $\alpha$ is strong in the above context, then the space of coboundaries $B_{\alpha}^{1}$ is closed.

Proof. We define a bijective map $\Phi$ from the coset space $\mathscr{U}(\mathscr{M}) / \mathscr{U}\left(\mathscr{M}^{\alpha}\right)$ to $Z_{\alpha}^{1}$ by $\Phi(v)=\left\{v \alpha_{g}\left(v^{*}\right)\right\}$. Because the metric defined by $L^{2}$-norm is translation invariant, $\mathscr{U}(\mathscr{M}) / \mathscr{U}\left(\mathscr{M}^{\alpha}\right)$ is a Polish space. We show that this $\Phi$ is a homeomorphism.

If $v_{n} \mathscr{U}\left(\mathscr{M}^{\alpha}\right) \rightarrow v \mathscr{U}\left(\mathscr{M}^{\alpha}\right)$ in the topology of the coset space, then there exists a sequence of unitaries $\left(w_{n}\right)$ in $\mathscr{U}\left(\mathscr{M}^{\alpha}\right)$ such that $\left\|v_{n} w_{n}-v\right\| \rightarrow 0$. This implies $\left\|v_{n} \alpha_{g}\left(v_{n}^{*}\right)-v \alpha_{g}\left(v^{*}\right)\right\|_{2} \rightarrow 0$, and hence $\Phi$ is continuous.

On the other hand, suppose $v_{n} \alpha_{g}\left(v_{n}^{*}\right) \rightarrow v \alpha\left(v^{*}\right)$. Then the sequence $\left(v^{*} v_{n}\right)$ is asymptotically $\alpha$-fixed; hence there exists a sequence $\left(w_{n}\right)$ in $\mathscr{M}^{\alpha}$ such that $\left\|v^{*} v_{n}-w_{n}\right\|_{2} \rightarrow 0$. We may assume each $w_{n}$ is a unitary by changing $w_{n}$ by a small operator if necessary, by Proposition 1.1.3 in Connes [C2]. Then the cosets $v_{n} \mathscr{U}\left(\mathscr{M}^{\alpha}\right)$ converge to $v \mathscr{U}\left(\mathscr{M}^{\alpha}\right)$, hence $\Phi^{-1}$ is also continuous. Thus there exists a positive number $\varepsilon_{n}$ such that $d\left(u \alpha_{g}\left(u^{*}\right), 1\right)<\varepsilon_{n}$ implies existence of $w \in \mathscr{U}\left(\mathscr{M}^{\alpha}\right)$ with $\|u w-1\|_{2}<1 / 2^{n}$. Suppose we have a sequence $\left(v_{n} \alpha_{g}\left(v_{n}^{*}\right)\right)$ converging to $u_{g}$ in $Z_{\alpha}^{1}$. Choosing a subsequence if necessary, we may assume $d\left(v_{n} \alpha_{g}\left(v_{n}^{*}\right), v_{n+1} \alpha_{g}\left(v_{n+1}^{*}\right)\right)<\varepsilon_{n}$. We choose a sequence of unitaries $\left(u_{n}\right)$ in $\mathscr{U}\left(\mathscr{M}^{\alpha}\right)$ by induction so that $\left\|v_{n} u_{n}-v_{n+1} u_{n+1}\right\|_{2}<$ $1 / 2^{n}$. Suppose $u_{1}, \ldots, u_{n}$ are chosen. Then there exists a unitary $w \in \mathscr{U}\left(\mathscr{M}^{\alpha}\right)$ such that $\left\|v_{n+1}^{*} v_{n} w_{n+1}-1\right\|_{2}<1 / 2^{n}$ by the definition of $\varepsilon_{n}$. Now set $u_{n+1}=u_{n} w_{n+1}$. The sequence $\left(v_{n} u_{n}\right)$ converges to some unitary $u \in \mathscr{M}$. We then have $u_{g}=v \alpha_{g}\left(v^{*}\right)$. 
Here we recall the definition of Kazhdan's property $\mathrm{T}$ for later use. A discrete group $G$ is said to have Kazhdan's property $\mathrm{T}$ if it has the following condition: There exists a finite subset $F \subset G$ and a positive number $\varepsilon$ such that for any unitary representation $U_{g}$ of $G$ on $H$, if there exists a vector $\xi \in \mathscr{H}$ such that $\|\xi\|=1$ and $\left\|U_{g} \xi-\xi\right\|<\varepsilon$ for all $g \in \mathscr{F}$, then there exists a non-zero vector $\eta \mathscr{H}$ such that $U_{g} \eta=\eta$ for all $g \in G$. (See Kazhdan [K] or Zimmer [Z2] for more about property T.)

We have the following characterization of Kazhdan's property $T$. Equivalence of (1), (2), and (3) are a non-commutative analogue of Theorem 3.2 in Schmidt [S]. Because $H_{\alpha}^{1}$ is not a group here, we consider all the cohomology classes in (3). (See Example 8.)

Theorem 2. Let $G$ be a discrete group. Then the following conditions are equivalent.

(1) $G$ has Kazhdan's property T.

(2) Any action of $G$ on a factor of type $\mathrm{II}_{1}$ is strong.

(3) For any action $\alpha$ of $G$ on a factor of type $\mathrm{II}_{1}$, each cohomology class is closed in $Z_{\alpha}^{1}$.

(4) For any action $\alpha$ of $G$ on a factor of type $\mathrm{II}_{1}$, the cohomology space $H_{\alpha}^{1}$ with the quotient topology is Hausdorff.

Proof. The equivalence of (1) and (2) was proved in Araki-Choda [ACh].

(2) $\Rightarrow(3)$ : Fix a unitary cocycle $u_{g}$ for $\alpha$. Because $\operatorname{Ad}\left(u_{g}\right)$. $\alpha_{g}$ is strong, we know that $\left\{v u_{g} \alpha_{g}\left(v^{*}\right) u_{g}^{*} \mid v \in \mathscr{U}(\mathscr{M})\right\}$ is closed by Proposition 1. Then it follows that $\left\{v u_{g} \alpha_{g}\left(v^{*}\right) \mid v \in \mathscr{U}(\mathscr{M})\right\}$ is closed.

(3) $\Rightarrow(4)$ : Because the metric in $Z_{\alpha}^{1}$ is invariant under the action of $\mathscr{U}(\mathscr{M})$, we have a metric on $H_{\alpha}^{1}$ and get the conclusion. (This is not just a pseudo-metric by closedness of each class.)

(4) $\Rightarrow(2)$ : If we define an action of $\mathscr{U}(\mathscr{M})$ on $Z_{\alpha}^{1}$ by $v \cdot u_{g}=$ $v u_{g} \alpha_{g}\left(v^{*}\right)$, then $\left(\mathscr{U}(\mathscr{M}), Z_{\alpha}^{1}\right)$ is a Polish transformation group in the sense of Effros [E]. The orbit space $H_{\alpha}^{1}$ is Hausdorff, hence $T_{0}$; thus Theorem 2.1 in [E] implies that $B_{1}^{\alpha}$ is homeomorphic to $\mathscr{U}(\mathscr{M}) / \mathscr{U}\left(\mathscr{M}^{\alpha}\right)$. Suppose a sequence $\left(x_{n}\right)$ in $\mathscr{M}$ is asymptotically $\alpha$ fixed. We show that the sequence is equivalent to another sequence in $\mathscr{M}^{\alpha}$. We may assume all $x_{n}$ 's are unitaries by a standard argument. Then the sequence $\left(x_{n} \alpha_{g}\left(x_{n}^{*}\right)\right)$ in $B_{\alpha}^{1}$ converges to 1 , and thus the sequence $\left(x_{n} \mathscr{U}\left(\mathscr{M}^{\alpha}\right)\right)$ converges to $\mathscr{U}\left(\mathscr{M}^{\alpha}\right)$ in $\mathscr{U}(\mathscr{M}) / \mathscr{U}\left(\mathscr{M}^{\alpha}\right)$. This shows (2) and completes the proof. 
Next we deal with openness of $B_{\alpha}^{1}$. The following technique is taken from Connes [C3]. This is also a non-commutative analogue of Theorem 3.4 (3) of Schmidt [S] and Theorem 2.11 of Zimmer [Z1].

Proposition 3. If $\alpha$ is an ergodic action of a discrete group $G$ with Kazhdan's property $\mathrm{T}$ on a factor $\mathscr{M}$ of type $\mathrm{II}_{1}$, then the set of coboundaries $B_{\alpha}^{1}$ is open in $Z_{\alpha}^{1}$.

Proof. Take a coboundary $u \alpha_{g}\left(u^{*}\right) \in Z_{\alpha}^{1}$. Let $F$ and $\varepsilon$ be as in the definition of Kazhdan's property T. Choose $\varepsilon_{0}$ so that $d\left(u \alpha_{g}\left(u^{*}\right), v_{g}\right)$ $<\varepsilon_{0}$ implies $\left\|u \alpha_{g}\left(u^{*}\right)-v_{g}\right\|_{2}<\varepsilon$ for all $g \in F$, and let

$$
\mathscr{V}=\left\{v_{g} \in Z_{\alpha}^{1} \mid d\left(u \alpha_{g}\left(u^{*}\right), v_{g}\right)<\varepsilon_{0}\right\} .
$$

It is enough that this neighborhood $\mathscr{V}$ of $u \alpha_{g}\left(u^{*}\right)$ is contained in the set of coboundaries. We assume $\mathscr{M}$ acts on the $L^{2}$-completion $\mathscr{H}$ of $\mathscr{M}$ by the left multiplication. Define a unitary representation $U_{g}$ of $G$ on $\mathscr{H}$ by $U_{g}\left(x \xi_{0}\right)=u \alpha_{g}\left(u^{*}\right) \alpha_{g}(x) v_{g}^{*} \xi_{0}$, where $\xi_{0}$ is the vector in $\mathscr{H}$ corresponding to $1 \in \mathscr{M}$. (The equality $U_{g h}=U_{g} U_{h}$ follows from the cocycle conditions of $v_{g}$.) We now have

$$
\left\|U_{g}\left(\xi_{0}\right)-\xi_{0}\right\|=\left\|u \alpha_{g}\left(u^{*}\right)-v_{g}\right\|_{2}<\varepsilon, \quad \text { for all } g \in F .
$$

Thus there exists a non-zero vector $\eta \in \mathscr{H}$ such that $U_{g}(\eta)=\eta$ for all $g \in G$. The operator $\eta \eta^{*}$ is well-defined and belongs to $L^{1}(\mathscr{M})_{+}$. This operator is fixed by $\operatorname{ad}(u) \cdot \alpha \cdot \operatorname{Ad}\left(u^{*}\right)$, which is ergodic. Thus $\eta$ is a unitary up to scalar, and we get a unitary $v$ such that $v=u \alpha_{g}\left(u^{*}\right) \alpha_{g}(v) v_{g}^{*}$, which means that the cocycle $v_{g}$ is a coboundary.

In Theorem 2 (3), we did not need an assumption on the action $\alpha$, but we assumed ergodicity of $\alpha$ in Proposition 3. We show that we cannot drop this assumption in general by the following example. The difference arose from the lack of the group structure of $Z_{\alpha}^{1}$.

EXAMPLE 4. Let $G$ be a discrete group with infinite conjugacy classes and Kazhdan's property T. (Take $G=\mathrm{SL}(3, Z)$, for instance.) Then consider the left regular representation $u_{g}$ of $G$, and let $\mathscr{R}(G)$ be the factor of type $\mathrm{II}_{1}$ generated by $u_{g}$ 's. Let $\mathscr{M}=\mathscr{R}(G) \bar{\otimes} \mathscr{R}(G)$, and $\alpha$ be the trivial action of $G$ on $\mathscr{M}$. Define

$$
v_{g}^{(n)}=u_{g} \otimes e_{n}+1 \otimes\left(1-e_{n}\right), \quad \text { for } n \in \mathbf{N}, g \in G,
$$

where $e_{n}$ is a projection in $\mathscr{R}(G)$ with the trace $1 / n$. Then these $v_{g}^{(n)}$ are cocycles for the trivial action $\alpha$, and $\left\|1-v_{g}^{(n)}\right\|-2 \leq 2 / \sqrt{n} \rightarrow 0$ 
as $n \rightarrow \infty$. But the set of coboundaries of $\alpha$ is just $\{1\}$. This shows that $B_{\alpha}^{1}$ is not open.

Thus, we concentrate on ergodic actions. We would like to show openness of each cohomology class for ergodic actions, but Proposition 3 does not imply it immediately because of lack of group structure in $Z_{\alpha}^{1}$. Indeed, cohomology classes are not open in general as Example 8 shows. For this reason, we consider only cocycles connecting ergodic actions. For this purpose, we show the following continuity first.

Proposition 5. Let $\alpha$ be an action of a discrete group $G$ with Kazhdan's property $\mathrm{T}$ on a factor $\mathscr{M}$ of type $\mathrm{II}_{1}$. Then the correspondence $u_{g} \in Z_{\alpha}^{1} \mapsto \mathscr{M}^{\operatorname{Ad}\left(u_{g}\right) \cdot \alpha_{g}}$ is uniformly continuous in the following sense: For any $\varepsilon_{0}>0$, there exists $\delta>0$ such that if $d\left(u_{g}, v_{g}\right)<\delta$, then

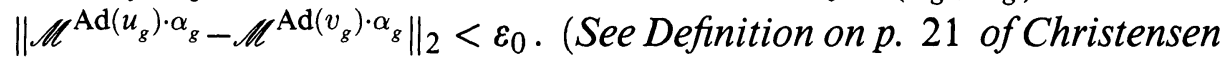
[Chr] for notation.)

Proof. Let $F \subset G$ and $\varepsilon>0$ be as in the definition of Kazhdan's property $\mathrm{T}$. Choose $\delta$ so that $d\left(u_{g}, v_{g}\right)<\delta$ implies $\left\|u_{g}-v_{g}\right\|<$ $\varepsilon \varepsilon_{0} / 2$ for all $g \in F$, and let $x$ be an element in $\mathscr{M}^{\mathrm{Ad}\left(v_{g}\right) \cdot \alpha_{g}}$ with $\|x\|_{\infty} \leq 1$. Set $\mathscr{N}=\mathscr{M}^{\operatorname{Ad}\left(u_{g}\right) \cdot \alpha_{g}}, y=\mathscr{E}_{\mathscr{N}}(x)$, and $z=x-y$, where $\mathscr{E}_{\mathcal{N}}$ is a conditional expectation onto $\mathscr{N}$.

By the GNS representation with respect to the trace $\tau$, we may assume $\mathscr{M}$ acts on the $L^{2}$-completion of $\mathscr{M}$ by the left multiplication. Let $\xi_{0}$ be the vector corresponding to $1 \leq \mathscr{M}$. We get a unitary representation $U_{g}$ of $G$ on this Hilbert space defined by $U_{g}\left(x \xi_{0}\right)=$ $\left(\operatorname{Ad}\left(u_{g}\right) \cdot \alpha_{g}(x)\right) \xi_{0}, x \in \mathscr{M}$. Then this $U_{g}$ restricts onto the orthogonal complement $\mathscr{H}$ of the $L^{2}$-completion of $\mathscr{N}$. Because we do not have a non-trivial invariant vector in $\mathscr{H}$ for $U_{g}$, we have an inequality

$$
\left\|\operatorname{Ad}\left(u_{g^{\prime}}\right) \cdot \alpha_{g^{\prime}}(x)-x\right\|_{2}=\left\|\operatorname{Ad}\left(u_{g^{\prime}}\right) \cdot \alpha_{g^{\prime}}(z)-z\right\|_{2} \geq \varepsilon\|z\|_{2},
$$

for some $g^{\prime} \in F$. Because

$$
\begin{aligned}
\left\|\operatorname{Ad}\left(u_{g^{\prime}}\right) \cdot \alpha_{g^{\prime}}(x)-x\right\|_{2} & =\left\|\operatorname{Ad}\left(u_{g^{\prime}} v_{g^{\prime}}^{*}\right)(x)-x\right\|_{2} \\
& \leq 2\left\|u_{g^{\prime}}-v_{g^{\prime}}\right\|_{2} \leq \varepsilon \varepsilon_{0},
\end{aligned}
$$

we get $\mathscr{M}^{\operatorname{Ad}\left(v_{g}\right) \cdot \alpha_{g}} \stackrel{\varepsilon_{0}}{\subset} \mathscr{N}$. By symmetry, we get the conclusion.

Now we work on ergodic actions of discrete groups. Let $\alpha$ be an ergodic action of a discrete group $G$ on a factor $\mathscr{M}$ of type $\mathrm{II}_{1}$. Define

$$
Z_{\alpha, \text { erg }}^{1}=\left\{u: G \rightarrow \mathscr{U}(\mathscr{M}) \mid u_{g} \in Z_{\alpha}^{1}, \operatorname{Ad}\left(u_{g}\right) \cdot \alpha_{g} \text { is also ergodic }\right\} \supset B_{\alpha}^{1},
$$


and $H_{\alpha, \text { erg }}^{1}=Z_{\alpha, \text { erg }}^{1} / \sim$. Consider the induced metric on $Z_{\alpha \text {, erg }}^{1}$. We assume now $G$ has Kazhdan's property $\mathrm{T}$ and $\alpha$ is ergodic in the rest of this section. Then we get the following immediately from Proposition 5.

Corollary 6. In the above context, $Z_{\alpha \text {, erg }}^{1}$ is closed in $Z_{\alpha}^{1}$.

Proof. The inequality $\|\mathscr{N}-\mathbf{C}\|_{2}<\delta$ for any $\delta>0$ implies $\mathscr{N}=$ C.

We show that property $\mathrm{T}$ implies the discreteness of the space $H_{\alpha, \text { erg }}^{1}$. The following proof is similar to that of Theorem 3. It implies the number of "different" cocycles connecting ergodic actions is "small". This is a rigidity result for cocycles.

THEOREM 7. Under the above context, $H_{\alpha, \text { erg }}^{1}$ is at most countable.

Proof. Since $H_{\alpha, \text { erg }}^{1}$ is separable, it is enough to show that each

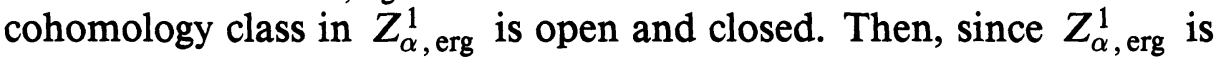
a disjoint of classes, it is sufficient to show that each class is open.

Take a cocycle $u_{g} \in Z_{\alpha \text {, erg }}^{1}$. Let $F$ and $\varepsilon$ be as in the definition of Kazhdan's property T. Choose $\varepsilon_{0}$ so that $d\left(u_{g}, v_{g}\right)<\varepsilon_{0}$ implies $\left\|u_{g}-v_{g}\right\|_{2}<\varepsilon$ for all $g \in F$, and let

$$
\mathscr{V}=\left\{v_{g} \in Z_{\alpha, \mathrm{erg}}^{1} \mid d\left(u_{g}, v_{g}\right)<\varepsilon_{0}\right\} .
$$

It is enough to show that this neighborhood $\mathscr{V}$ of $u_{g}$ is contained in the class of $u_{g}$. We assume $\mathscr{M}$ acts on the $L^{2}$-completion $\mathscr{H}$ of $\mathscr{M}$ by the left multiplication. Define a unitary representation $U_{g}$ of $G$ on $\mathscr{H}$ by $U_{g}\left(x \xi_{0}\right)=u_{g} \alpha_{g}(x) v_{g}^{*} \xi_{0}$, where $\xi_{0}$ is the vector in $\mathscr{H}$ corresponding to $1 \in \mathscr{M}$. (The equality $U_{g h}=U_{g} U_{h}$ follows from the cocycle conditions of $u_{g}$ and $\left.v_{g}.\right)$ We now have $\left\|U_{g}\left(\xi_{0}\right)-\xi_{0}\right\|=$ $\left\|u_{g}-v_{g}\right\|_{2}<\varepsilon$ for all $g \in F$. Thus there exists a non-zero vector $\eta \in \mathscr{H}$ such that $U_{g}(\eta)=\eta$ for all $g \in G$. The operator $\eta^{*} \eta$ is welldefined and belongs to $L^{1}(\mathscr{M})_{+}$. This operator is fixed by $\operatorname{Ad}\left(v_{g}\right) \cdot \alpha_{g}$, which is ergodic. Thus $\eta$ is a unitary up to scalar, and we get a unitary $v$ such that $v=u_{g} \alpha_{g}(v) v_{g}^{*}$, which means $u_{g} \sim v_{g}$.

A direct analogue of Theorem 3.4 (3) of Schmidt [S] and Theorem 2.11 of Zimmer [Z1] would be that each cohomology class of an ergodic action of a discrete group with Kazhdan's property $\mathrm{T}$ would be open. But this statement is invalid in general as the following example, similar to Example 4, shows. This justifies that we considered only $Z_{\alpha, \text { erg }}^{1}$ in the above theorem. 
EXAMPle 8. Let $G, u_{g}, \mathscr{M}$ be as in Example 4. Let $\alpha$ be the action of $G \times G$ on $\mathscr{M}$ defined by $\alpha_{(g, h)}=\operatorname{Ad}\left(u_{g} \otimes u_{h}\right)$ for $g, h \in G$. Note that $G \times G$ also has Kazhdan's property T (see Connes [C3]), and this action $\alpha$ is ergodic. Define $v_{(g, h)}=u_{g}^{*} \otimes u_{h}^{*}$ and

$$
v_{(g, h)}^{(n)}=1 \otimes\left(e_{n} u_{h}^{*}\right)+u_{g}^{*} \otimes\left(1-e_{n}\right) u_{h}^{*}, \quad n \in \mathbf{N}, g, h \in G,
$$

where $e_{n}$ is a projection in $\mathscr{R}(G)$ with the trace $1 / n$. An easy computation shows that these are $\alpha$-cocycles. Then $\left\|v_{(g, h)}-v_{(g, h)}^{(n)}\right\|_{2} \leq$ $2 / \sqrt{n} \rightarrow 0$ as $n \rightarrow \infty$. If we have $v_{(g, h)} \sim v_{(g, h)}^{(n)}$ for some $n$, then it implies $v_{(g, h)}=v_{(g, h)}^{(n)}$, which is a contradiction. This show that the cohomology class of $v_{(g, h)}$ is not open.

If we have "too many" ergodic actions compared to cocycles, then it means the number of cocycle conjugacy classes is large. Jones [J1] first showed that a discrete group with Kazhdan's property $\mathrm{T}$ has two mutually non-cocycle conjugate free actions on the AFD factor of type $\mathrm{II}_{1}$. M. Choda [Ch1, Ch2] constructed a continuous family of mutually nonconjugate ergodic free actions of $\operatorname{SL}(n, \mathbf{Z}), n \geq 3$, and $\mathrm{Sp}(n, \mathbf{Z}), n \geq 2$, on the AFD factor $\mathscr{R}$ of type $\mathrm{II}_{1}$, and she asked in the first question of page 534 of [Ch2] whether a discrete group with Kazhdan's property $\mathrm{T}$ has two non-cocycle conjugate ergodic free actions on the AFD factor of type $\mathrm{II}_{1}$, and obtained an affirmative answer in it by constructing two different closed product algebras. We get the following corollary about this question. It shows that these groups have the totally opposite property of amenable groups with respect to free actions on the AFD factor of type $\mathrm{II}_{1}$. (Theorem 2.7 in Ocneanu [0] asserts that every discrete amenable group has the unique free action, up to cocycle conjugacy, on the AFD factor $\mathscr{R}$ of type $\mathrm{II}_{1}$. )

COROLlary 9. Each of the groups $\operatorname{SL}(n, \mathbf{Z}), n \geq 3$, and $\operatorname{Sp}(n, \mathbf{Z})$, $n \geq 2$, has a continuous family of ergodic free actions on the AFD factor of type $\mathrm{II}_{1}$ such that any two of them are not cocycle conjugate.

Proof. Suppose the number of different cocycle conjugacy classes of actions in the family M. Choda constructed is countable. Then at least one class contains continuously many actions. Let $\alpha$ be an action in this class. Since the other actions in this class are ergodic and cocycle conjugate to $\alpha$, there exist continuously many cocycles such that any two of them are not cohomologous, hence $H_{\alpha \text {, erg for }}^{1}$ this $\alpha$ is uncountable, which is a contradiction. 
REMARK 10. All the above actions are ergodic, hence ergodic at infinity in the sense of Jones [J2]. Thus any two of them cannot be distinguished by the method of Jones [J2]. All the crossed product algebras of the above actions have property $\mathrm{T}$ as shown in Choda [Ch2].

2. Entropy and cohomology for amenable group actions. In this section, we study what follows from Ocneanu's work [0] about cohomological properties of free actions of discrete amenable groups on the AFD factor $\mathscr{R}$ of type $\mathrm{II}_{1}$. In Proposition 7.2 in Ocneanu [O], he obtained 1-cohomology vanishing in the ultraproduct algebra. His method appeals to Shapiro's lemma type argument based on his noncommutative Rohlin theorem. If we go back to the original algebra, we get the following by his method.

Proposition 11. Let $\alpha$ be a free action of a discrete amenable group $G$ on the AFD factor $\mathscr{R}$ of type $\mathrm{II}_{1}$. Then $B_{\alpha}^{1}$ is dense in $Z_{\alpha}^{1}$.

Proof. Apply the proof of Proposition 7.2 in [O] to a given cocycle $v_{g}$. Though $v_{g}$ is not in the ultraproduct, the proof works until line 11 of page 63 if we think $w$ in the proof is an element of $\mathscr{M}^{\omega}$, because we have a non-commutative Rohlin Theorem for our $\alpha$. Then $v_{g}$ is equal to a coboundary $w \alpha_{g}\left(w^{*}\right)$ in the ultraproduct algebra with a small error. Then choosing a unitary $u_{n}$ form the sequence representing $w$ in the proof, we get a coboundary $u_{n} \alpha_{g}\left(u_{n}^{*}\right)$ such that $\left\|u_{n} \alpha_{g}\left(u_{n}^{*}\right)-v_{g}\right\|_{1}<1 / n$ for all $g \in F_{n}$, where $F_{n}$ is an increasing sequence of finite subsets of $G$ with $\bigcup_{n} F_{n}=G$. This shows the desired density.

We consider the number of unitary cocycles next. As in Choda's result used in $\S 1$, we would like to obtain a large number of conjugacy classes of ergodic free actions. In order to distinguish general discrete amenable group actions, we extend Connes-Størmer entropy in [CS] to countable amenable group actions. It will give us continuous conjugacy classes of non-commutative Bernoulli shifts. (See Ornstein-Weiss [OW] for entropy of group actions on probability spaces.)

For reference, we list basic definitions and properties of ConnesStørmer entropy from [CS].

[DEFINITON 1 of [CS]]. For finite dimensional von Neumann subalgebras $N_{1}, \ldots, N_{k}$ of $\mathscr{R}$, define

$$
H\left(N_{1}, \ldots, N_{k}\right)=\sup _{x \in S_{k}}\left(\sum \eta\left(\tau\left(x_{i_{1}}, \ldots, i_{k}\right)\right)-\sum \tau\left(\eta\left(E_{N_{l}}\left(x_{i_{l}}^{l}\right)\right)\right)\right),
$$


where $\eta(x)=-x \log x, S_{k}$ is the set of all families $\left(x_{i_{1}}, \ldots, i_{k}\right), i_{j} \in \mathbf{N}$, of positive elements of $\mathscr{R}$, zero except for a finite number of indices, and with the sum equal to 1 , and

$$
x_{i_{l}}^{l}=\sum_{i_{1}, \ldots, i_{l-1}, i_{l+1}, \ldots, i_{k}} x_{i_{1}, \ldots, i_{k}} .
$$

[Properties of $H$ ].

$$
H\left(N_{1}, \ldots, N_{k}\right) \leq H\left(P_{1}, \ldots, P_{k}\right) \text { when } N_{j} \subset P_{j},
$$

(B)

$$
\begin{aligned}
H\left(N_{1}, \ldots, N_{p}\right) & \leq H\left(N_{1}, \ldots, N_{k}\right)+H\left(N_{k+1}, \ldots, N_{p}\right), \\
P_{1}, \ldots, P_{n} & \subset P \Rightarrow H\left(P_{1}, \ldots, P_{n}, P_{n+1}, \ldots, P_{m}\right) \\
& \leq H\left(P, P_{n+1}, \ldots, P_{m}\right)
\end{aligned}
$$

(D) $\quad H(N)=\sum \eta\left(\tau\left(e_{\alpha}\right)\right)$, when $\sum e_{\alpha}=1, e_{\alpha}:$ minimial,

$$
H\left(N_{1}, \ldots, N_{k}\right)=H\left(\left(N_{1} \cup \cdots \cup \mathbf{N}_{k}\right)^{\prime \prime}\right),
$$

when $\left(N_{1} \cup \cdots \cup N_{k}\right)^{\prime \prime}$ is generated by pairwise commuting subalgebras $P_{j} \subset N_{j}$,

$$
\begin{aligned}
& H\left(N_{1}, \ldots, N_{k}\right) \leq H\left(P_{1}, \ldots, P_{k}\right)+\sum H\left(N_{j} \mid P_{j}\right) \\
& \quad \text { where } H(N \mid P)=\sup _{x \in S_{1}} \sum\left(\tau\left(\eta\left(E_{P}\left(x_{i}\right)\right)\right)-\tau\left(\eta\left(E_{N}\left(x_{i}\right)\right)\right)\right) .
\end{aligned}
$$

[TheOREM 1 OF [CS]]. For each $n \in \mathbf{N}$ and $\varepsilon>0$, there is $a \delta>0$ such that for any pair of von Neumann subalgebras $N, P \subset \mathscr{R}$, the conditions $\operatorname{dim} N=n$ and $N \stackrel{\delta}{\subset} P$ imply $H(N \mid P)<\varepsilon$.

Let $G$ be a countable amenable group. Choose an increasing sequence of finite subsets $\left\{A_{n}\right\}$ by amenability such that

$$
\lim _{n \rightarrow \infty} \frac{\left|g A_{n} \Delta A_{n}\right|}{\left|A_{n}\right|}=0, \quad \text { for all } g \in G \text {. }
$$

(It is well known that existence of such a sequence is equivalent to amenability. See Greenleaf [G] for instance.)

For an action $\alpha$ of $G$ on a factor $\mathscr{M}$ of type $\mathrm{II}_{1}$, we define

$$
H(N, \alpha, A)=H\left(\alpha_{g_{1}}(N), \ldots, \alpha_{g_{m}}(N)\right),
$$

$$
\text { where } A=\left\{g_{1}, \ldots, g_{m}\right\} \subset G \text {, }
$$




$$
\begin{gathered}
H(N, \alpha)=\limsup _{n \rightarrow \infty} \frac{1}{\left|A_{n}\right|} H\left(N, \alpha, A_{n}\right), \\
H(\alpha)=\sup _{\substack{N \subset \mathscr{M}, \\
\text { finite dimensional }}} H(N, \alpha) .
\end{gathered}
$$

Note that $H(N, \alpha) \leq H(N)$ by property (B) in Connes-Størmer [CS]. The sup in the third definition may be infinity.

The following is the non-commutative Kolmogorov-Sinai theorem and an analogue of Theorem 2 in [CS].

Proposition 12. Let $P_{q}, q \geq 1$, be an increasing sequence of $f-$ nite dimensional subalgebras of the AFD factor $\mathscr{R}$ of type $\mathrm{II}_{1}$ with $\overline{\bigcup_{q=1}^{\infty} P_{q}}=\mathscr{R}$. Then

$$
H(\alpha)=\lim _{q \rightarrow \infty} H\left(P_{q}, \alpha\right)
$$

Proof. The same proof as that of Theorem 2 in [CS] works. (Use property (F) and Theorem 1 in [CS] to get $H(N, \alpha) \leq H\left(P_{q}, \alpha\right)+\varepsilon$ for given $\varepsilon$.)

The following is a computation of entropy for Bernoulli shifts and corresponds to Theorem 3 in [CS].

Proposition 13. Represent the AFD factor $\mathscr{R}$ of type $\mathrm{II}_{1}$ as the infinite tensor product of $k$-dimensional matrix algebra $M_{k}(\mathbf{C})$ with respect to the trace over a countable amenable group $G$. Define an action $\alpha_{g}$ of $G$ on $\mathscr{R}$ by the Bernoulli shift. Then we get $H(\alpha)=$ $\log k$.

Proof. For $q \geq 1$, define

$$
P_{q}=\bigotimes_{g \in A_{q}} M_{k}(\mathbf{C}) \otimes \bigotimes_{g \notin A_{q}} \mathbf{C}
$$

It is clear that $P_{q}$ is an increasing sequence of finite dimensional algebras in $\mathscr{R}$ and $\bigcup_{q=1}^{\infty} P_{q}$ is weakly dense in $\mathscr{R}$. Thus we can apply Proposition 12, and get

$$
\begin{aligned}
H(\alpha) & =\lim _{q \rightarrow \infty} \lim _{n \rightarrow \infty} \frac{1}{\left|A_{n}\right|} H\left(P_{q}, \alpha, A_{n}\right) \\
& =\lim _{q \rightarrow \infty} \lim _{n \rightarrow \infty} \frac{\left|A_{q} A_{n}\right|}{\left|A_{n}\right|} \log k \\
& =\log k,
\end{aligned}
$$

by the definition of $A_{n}$. 
The following shows that this entropy for group actions is more powerful than entropy of single automorphisms.

EXAMPLE 14. Let $G=\mathbf{Z}^{2}$ and apply the above construction for $k=2,3$ to get actions $\alpha$ and $\beta$ of $\mathbf{Z}^{2}$ on $\mathscr{R}$. Then for any $g \in \mathbf{Z}^{2}$, $g \neq 0, \alpha_{g}$ and $\beta_{g}$ have the entropy infinity as single automorphisms. Actually, they are both conjugate to the shift on $\bigotimes_{\mathrm{Z}} \mathscr{R} \cong \mathscr{R}$. But as group actions, they have different entropy, and thus they are nonconjugate.

Let $G=\mathbf{Z}[1 / 2] / Z$ and apply the above construction for $k=2,3$ to get actions $\alpha$ and $\beta$ of $\mathbf{Z}[1 / 2] / Z$ on $\mathscr{R}$. Then for any $g \in$ $\mathbf{Z}[1 / 2] / \mathbf{Z}, \alpha_{g}$ and $\beta_{g}$ have the entropy zero as single automorphism because they are both periodic, by Remark 6 of Connes-Størmer [CS]. But as group actions, they have different entropy, and thus they are non-conjugate.

We would like to get continuously many values of the entropy, so we introduce the following as in Theorem 4 in [CS].

Let $\mathscr{M}$ be the infinite tensor product of $M_{k}(\mathbf{C})$ with respect to the product state $\psi^{\lambda}=\otimes \varphi^{\lambda}, \lambda=\left(\lambda_{1}, \ldots, \lambda_{k}\right)$, where $\varphi^{\lambda}$ on $M_{k}(\mathbf{C})$ is defined by

$$
\varphi^{\lambda}(x)=\operatorname{Tr}\left(x \cdot\left(\begin{array}{ccc}
\lambda_{1} & & \\
& \ddots & \\
& & \lambda_{k}
\end{array}\right)\right), \quad \lambda_{1}+\cdots+\lambda_{k}=1, \lambda_{j}>0 .
$$

Let $\beta_{g}^{\lambda}$ be the ergodic action of a countable amenable group $G$ on $\mathscr{M}$ given by the Bernoulli shift. The centralizer $\mathscr{M}_{\psi^{\lambda}}$ is isomorphic to $\mathscr{R}$ as in the proof of Theorem 4 of Connes-Størmer [CS]. Define the action $\alpha_{g}^{\lambda}$ to be the restriction of $\beta_{g}^{\lambda}$ on $\mathscr{M}_{\psi^{\lambda}}$. This is also ergodic, and this is a free action. We use entropy as a conjugacy invariant to distinguish these actions. by

Proposition 15. The entropy $H\left(\alpha^{\lambda}\right)$ of the above action $\alpha_{g}^{\lambda}$ is given

$$
H\left(\alpha^{\lambda}\right)=\sum_{j=1}^{k}-\lambda_{j} \log \lambda_{j}
$$

Proof. Define

$$
F_{p}=\left(\bigotimes_{g \in A_{p}}\left(M_{k}(\mathbf{C}), \varphi^{\lambda}\right)\right)_{\bigotimes \varphi^{\lambda}} ; \quad D_{p}=\bigotimes_{g \in A_{p}}\left(\begin{array}{ll}
* & 0 \\
0 & *
\end{array}\right) .
$$


Then using Properties (C), (D), (E) and (C) of Connes-Stø̣rmer [CS], we get

$$
\begin{aligned}
H\left(F_{p}, \alpha, A_{n}\right) & \leq H\left(\left(\bigotimes_{g \in A_{p} A_{n}}\left(M_{k}(\mathbf{C}), \varphi^{\lambda}\right)\right)_{\bigotimes \varphi^{\lambda}}\right) \\
& =\left|A_{p} A_{n}\right| \sum_{j=1}^{k}-\lambda_{j} \log \lambda_{j} \\
& =H\left(D_{p}, \alpha, A_{n}\right) \\
& \leq H\left(F_{p}, \alpha, A_{n}\right),
\end{aligned}
$$

as in the computation in p. 304 of [CS]. Thus we have

$$
\begin{aligned}
H\left(\alpha^{\lambda}\right) & =\lim _{p} \lim _{n} \frac{1}{\left|A_{n}\right|} H\left(F_{p}, \alpha, A_{n}\right) \\
& =\sum_{j=1}^{k}-\lambda_{j} \log \lambda_{j} .
\end{aligned}
$$

as in the proof of Proposition 13.

THEOREM 16. For a free action $\alpha$ of a discrete amenable group $G$ on the AFD factor $\mathscr{R}$ of type $\mathrm{II}_{1}, H_{\alpha}^{1}$ is uncountable. For an ergodic free action $\alpha$ of $G$ on $\mathscr{R}, H_{\alpha, \mathrm{erg}}^{1}$ is uncountable.

Proof. By Proposition 15, we know that $G$ has a continuous family of mutually nonconjugate ergodic free actions on $\mathscr{R}$. By Ocneanu's result [O, Theorem 2.7], its members are all cocycle conjugate. Thus we get the conclusion.

Now we get the following. This is a von Neumann algebra analogue of Schmidt [S, Remark 3.5].

Theorem 1.7. Let $G$ be a countable group. Then the following conditions are equivalent.

(1) $G$ is amenable.

(2) No free action $\alpha$ of $G$ on the AFD factor $\mathscr{R}$ of type $\mathrm{II}_{1}$ is strong.

(3) $B_{\alpha}^{1} \subsetneq \overline{B_{\alpha}^{1}}=Z_{\alpha}^{1}$ for all free actions $a$ of $G$ on the AFD factor $\mathscr{R}$ of type $\mathrm{II}_{1}$.

Proof. (2) $\Rightarrow(1)$. If $G$ is not amenable, then $G$ has a strongly ergodic free action $\alpha$ on $\mathscr{R}$ obtained by the Bernoulli shift as in Jones [J2]. 
$(1) \Rightarrow(3)$ : If $G$ is amenable, we get the conclusion by Proposition 11 and Theorem 16.

(3) $\Rightarrow(2)$ : This follows from Proposition 1 .

Added in Proof. After the submission of this paper, the author received the following related paper.

S. Popa, "Some rigidity results in type II, factors".

The author thanks Professor Popa for calling attention to this preprint.

\section{REFERENCES}

[ACh] H. Araki and M. Choda, Property $T$ and actions on the hyperfinite $\mathrm{II}_{1}$-factor, Math. Japon., 28 (1983), 205-209.

[Ch1] M. Choda, $A$ continuum of non-conjugate property $T$ actions of $\operatorname{SL}(n, \mathbf{Z})$ on the hyperfinite $\mathrm{II}_{1}$-factor, Math. Japon., 30 (1985), 133-150.

[Ch2] _ Outer actions of groups with property $T$ on the hyperfinite $\mathrm{II}_{1}$-factor, Math. Japon., 31 (1986), 533-551.

[Chr] E. Christensen, Subalgebras of a finite algebra, Math. Ann., 243 (1979), 1729.

[C1] A. Connes, almost periodic states and factors of type $\mathrm{III}_{1}$, J. Funct. Anal., 16 (1974), 415-445.

[C2] _ Outer conjugacy classes of automorphisms of factors, Ann. Sci. École Norm. Sup., 8 (1975), 383-419.

[C3] _ A factor of type $\mathrm{II}_{1}$ with countable fundamental group, J. Operator Theory, 4 (1980), 151-153.

[CJ1] A. Connes and V. F. R. Jones, $A \mathrm{II}_{1}$ factor with two nonconjugate Cartan subalgebras, Bull. Amer. Math. Soc., 6 (1982), 211-212.

[CJ2] _ Property $T$ for von Neumann algebras, Bull. London Math. Soc., 17 (1985), 57-62.

[CS] A. Connes and E. Størmer, Entropy for automorphisms of $\mathrm{II}_{1}$ von Neumann algebras, Acta Math., 134 (1975), 289-306.

[CW] A. Connes and B. Weiss, Property $T$ and asymptotically invariant sequences, Israel J. Math., 37 (1980), 209-210.

[E] E. G. Effros, Transformation groups and $C^{*}$-algebras, Ann. Math., 81 (1965), 38-55.

[G] F. P. Greenleaf, Invariant Means on Topological Groups and Their Applications, van Nostrand, New York, 1969.

[J1] V. F. R. Jones, Actions of discrete groups on factors, in Operator algebras and applications, Part 2 (Kingston, Ont., 1980), Proc. Sympos. Pure Math. Vol. 38, Amer. Math. Soc., Providence, R.I., 1982, pp. 167-177.

[J2] - A converse to Ocneanu's theorem, J. Operator Theory, 10 (1983), 61-63.

[K] D. Kazhdan, Connection of the dual space of a group with the structure of its : closed subgroups, Funct. Anal. Appl., 1 (1967), 63-65.

[O] A. Ocneanu, Actions of discrete amenable groups on factors, Lecture Notes in Math. No. 1138, Springer, Berlin, 1985.

[OW] D. Ornstein and B. Weiss, Entropy and isomorphism theorems for actions of amenable groups, J. Analyse Math., 48 (1987), 1-141. 
[S] K. Schmidt, Amenability, Kazhdan's property $T$, strong ergodicity and invariant means for ergodic group-actions, Ergodic Theory Dynamical Systems, 1 (1981), 223-226.

[Z1] R. J. Zimmer, On the cohomology of ergodic actions of semisimple Lie groups and discrete subgroups, Amer. J. Math., 103 (1981), 937-950.

[Z2] _ Ergodic Theory and Semisimple Groups, Birkhäuser, 1984.

Received January 4, 1990.

UNIVERSITY OF TOKYO

HONGO, TOKYO, 113 JAPAN 



\section{PACIFIC JOURNAL OF MATHEMATICS EDITORS}

\author{
V. S. VARAdaraJan \\ (Managing Editor) \\ University of California \\ Los Angeles, CA 90024-1555-05 \\ Herbert Clemens \\ University of Utah \\ Salt Lake City, UT 84112 \\ THOMAS ENRIGHT \\ University of California, San Diego \\ La Jolla, CA 92093
}

R. FINN

Stanford University

Stanford, CA 94305

Hermann FlaschKa

University of Arizona

Tucson, AZ 85721

VAUGHAN F. R. Jones

University of California

Berkeley, CA 94720

STEVEN KerckHofF

Stanford University

Stanford, CA 94305

\section{C. MOORE}

University of California

Berkeley, CA 94720

Martin ScharlemanN

University of California

Santa Barbara, CA 93106

HAROLd STARK

University of California, San Diego

La Jolla, CA 92093
R. ARENS
E. F. BECKENBACH
F. WoLF
K. YoshidA
ASSOCIATE EDITORS

\begin{tabular}{ll}
\multicolumn{1}{c}{$(1906-1982)$} & \multicolumn{1}{c}{$(1904-1989)$} \\
SUPPORTING & INSTITUTIONS \\
UNIVERSITY OF ARIZONA & UNIVERSITY OF OREGON \\
UNIVERSITY OF BRITISH COLUMBIA & UNIVERSITY OF SOUTHERN CALIFORNIA \\
CALIFORNIA INSTITUTE OF TECHNOLOGY & STANFORD UNIVERSITY \\
UNIVERSITY OF CALIFORNIA & UNIVERSITY OF HAWAII \\
MONTANA STATE UNIVERSITY & UNIVERSITY OF TOKYO \\
UNIVERSITY OF NEVADA, RENO & UNIVERSITY OF UTAH \\
NEW MEXICO STATE UNIVERSITY & WASHINGTON STATE UNIVERSITY \\
OREGON STATE UNIVERSITY & UNIVERSITY OF WASHINGTON
\end{tabular}

The Supporting Institutions listed above contribute to the cost of publication of this Journal, but they are not owners or publishers and have no responsibility for its content or policies.

Mathematical papers intended for publication in the Pacific Journal of Mathematics should be in typed form or offset-reproduced (not dittoed), double spaced with large margins. Please do not use built up fractions in the text of the manuscript. However, you may use them in the displayed equations. Underline Greek letters in red, German in green, and script in blue. The first paragraph must be capable of being used separately as a synopsis of the entire paper. In particular it should contain no bibliographic references. Please propose a heading for the odd numbered pages of less than 35 characters. Manuscripts, in triplicate, may be sent to any one of the editors. Please classify according to the 1991 Mathematics Subject Classification scheme which can be found in the December index volumes of Mathematical Reviews. Supply name and address of author to whom proofs should be sent. All other communications should be addressed to the managing editor, or Elaine Barth, University of California, Los Angeles, California 90024-1555-05.

There are page-charges associated with articles appearing in the Pacific Journal of Mathematics. These charges are expected to be paid by the author's University, Government Agency or Company. If the author or authors do not have access to such Institutional support these charges are waived. Single authors will receive 50 free reprints; joint authors will receive a total of 100 free reprints. Additional copies may be obtained at cost in multiples of 50 .

The Pacific Journal of Mathematics (ISSN 0030-8730) is published monthly except for July and August. Regular subscription rate: $\$ 190.00$ a year (10 issues). Special rate: $\$ 95.00$ a year to individual members of supporting institutions.

Subscriptions, orders for numbers issued in the last three calendar years, and changes of address should be sent to Pacific Journal of Mathematics, P.O. Box 969, Carmel Valley, CA 93924, U.S.A. Old back numbers obtainable from Kraus Periodicals Co., Route 100, Millwood, NY 10546.

The Pacific Journal of Mathematics at P.O. Box 969, Carmel Valley, CA 93924 (ISSN 0030-8730) is published monthly except for July and August. Second-class postage paid at Carmel Valley, California 93924, and additional mailing offices. Postmaster: send address changes to Pacific Journal of Mathematics, P.O. Box 969, Carmel Valley, CA 93924.

PUBLISHED BY PACIFIC JOURNAL OF MATHEMATICS, A NON-PROFIT CORPORATION Copyright (C) 1991 by Pacific Journal of Mathematics 


\section{Pacific Journal of Mathematics}

Vol. 149, No. $2 \quad$ June, 1991

Manuel Alfaro Garcia, Mark Conger and Kenneth Hodges, The structure

of singularities in $\Phi$-minimizing networks in $\mathbf{R}^{2}$

Werner Balser, Dependence of differential equations upon parameters in

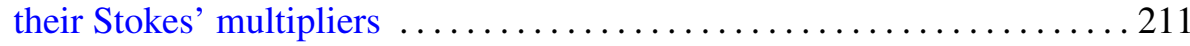

Enrico Casadio Tarabusi and Stefano Trapani, Envelopes of holomorphy

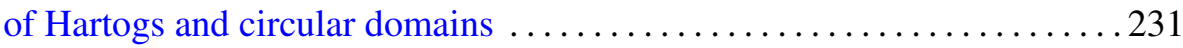

Hermann Flaschka and Luc Haine, Torus orbits in $G / P \ldots \ldots \ldots \ldots 251$

Gyo Taek Jin, The Cochran sequences of semi-boundary links .......... 293

Yasuyuki Kawahigashi, Cohomology of actions of discrete groups on

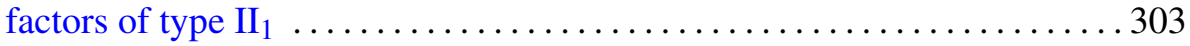

Ki Hyoung Ko and Lawrence Smolinsky, A combinatorial matrix in 3-manifold theory

W. B. Raymond Lickorish, Invariants for 3-manifolds from the combinatorics of the Jones polynomial

Peter Arnold Linnell, Zero divisors and group von Neumann algebras . . . . . 349

Bruce Harvey Wagner, Classification of essential commutants of abelian

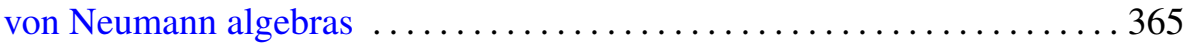

Herbert Walum, Multiplication formulae for periodic functions ..........383 